\title{
مدى تضمين مهارات القرن الحادي والعشرين في مقررات الكيمياء بالمرحلة
}

\section{الثانوية في السودان}

سيف الدين إدريس أونيا1

أبكر يعقوب آدم لقمان

كلية التربية، جامعة الخرطوم

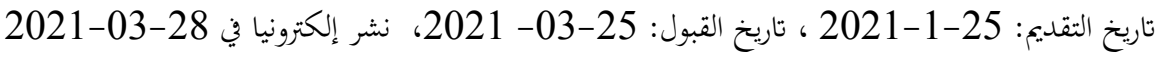
https://doi.org/10.36602/faj/2021.n.17.09

الملتخص:

هدفت الدراسة إلى التعرف على مدى تضمين مهارات القرن الحادي والعشرين في مقررات الكيمياء بالمرحلة الثانوية في السودان، ولتحقيق هذا الهدف استخدم الباحثان المنهج الوصفي التحليلي باستخدام استمارة تحليل المحتوى معدلة من قبل الباحث، حيث اشتملت الأداة في

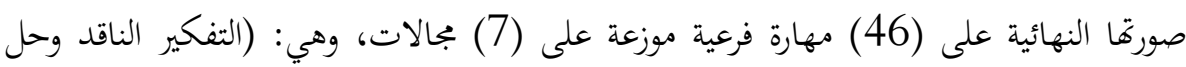

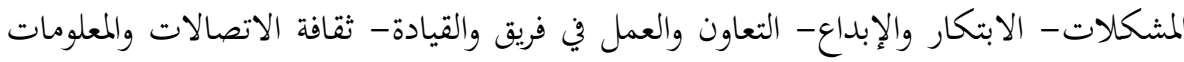
والإعلام- ثقافة الحوسبة وتقنية المعلومات والاتصال- المهنة والتعلم المعتمد على الذات- فهم

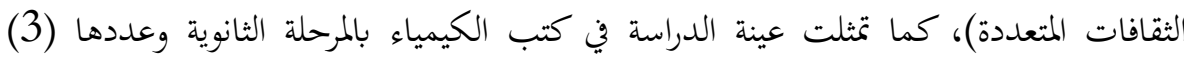
كتب. ولتحليل البيانات تم استخدام (التكرارات- النسب المئوية)، وأظهرت النتائج الخفاض

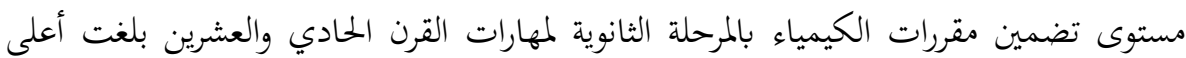
نسبة (44,2\%) وأدنى نسبة تضمين بلغت (1,5\%)، وفي ضوراء النتائج أوصى الباحث ضرورة توجيه عناية واهتمام القائمين على تخطيط المناهج وإعدادها في دمج مهارات القرن الحادي والعشرين في المقررات الدراسية لجميع المراحل التعليمية، وأيضاً وضع معايير لبناء مناهج الكيمياء

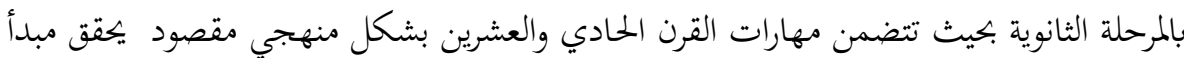
التكامل والاستمرارية. الكلمات المفتاحية: مهارات القرن الحادي والعشرين، تحليل المحتوى، مقررات الكيمياء، المرحلة الثانوية.

saifdldom07@gmail.com ${ }^{1}$ 


\title{
The extent to which $21^{\text {st }}$ Century Skills are included in high School Chemistry courses in Sudan
}

\section{Abaker Yagoub Adam}

Saifaldin Idris Onia

Faculty of Education, University of Khartoum

\begin{abstract}
:
The study aimed to identify the extent to which $21^{\text {st }}$ century skills are included in chemistry courses at the secondary level in Sudan, to achieve this goal the researcher used the descriptive analytical method using the content analysis from modified by the researcher as the tool included in its final form 46 sub- skills on 7 areas which are(critical thinking and problem solving, innovative and creativity, cooperation and team work and leadership communication technology information and media computing technology, profession self - reliant learning, understanding multiple culture). The study was also represented in the chemistry courses to first, second, and third grades. To analyze the data frequencies and percentages were used. The results showed a low level of inclusion chemistry courses at the secondary stage for the twenty first century skills reached $44,2 \%$ and the lowest inclusion rate reached $1,5 \%$. The researchers recommended the need to direct the care and attention of those involved in curriculum planning and preparation in the approach to $21^{\text {st }}$ century skills in study designs for all educational levels, also setting standards for building high school chemistry curricula to include 21 st century skills as a systematic manner that achieves the principal of complementarity continuity.
\end{abstract}

Keywords: $21^{\text {st }}$ Century Skills, Content Analysis, Chemistry Courses, Secondary Stag

1

$$
\begin{aligned}
& \text { يشهد العالم تطورات علمية في مختلف المجالات وقد تركت هذه التطورات بصماتا } \\
& \text { على مختلف مناحي الحياة، ومنها التعليم، فنادى التربويين بضرورة الإفادة من مستجدات } \\
& \text { العلم في النهوض بالعمل التربوي وتطويره، وكانت وسيلة التربويين لإجراء التغيير المنشود هي }
\end{aligned}
$$


المنهج المدرسي بما يتضمنه من معارف ومهارات واتحاهات وقيم تتفق وخصائص المتعلم،

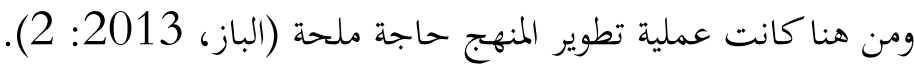
حيث شهدت الفترة الأخيرة من القرن الحادي والعشرين اهتماماً واسعاً بالمهارات الضرورية والكافية للنجاح في الحياة والعمل، لذا أصبحت الحاجة إلى التغيير والتحديث في برامج التربية العلمية ملحة وحتمية لتلبية متطلبات القرن الحادي والعشرين، واستجابة لذلك سعت كثير من المؤسسات والمنظمات التربوية إلى تحديد مهارات القرن الحادي والعشرين، وصياغة أطر وأفكار لدمج هذه المهارات وتكاملها مع النظم التعليمية، التي تعين المتعلم على التكيف مع العالم المتغير، ومواكبة التغييرات المتلاحقة التي يتسم بها، كما تساعده على

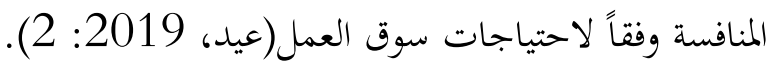

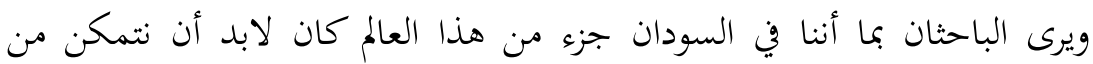
مواكبة هذه التغييرات وتحقيق تناغم إيجابي معها يستدعي مزيداً من الجهود المنظمة، والمركزة من المؤسسات التربوية التي تتطلع إلى مواكبة التغيير وقيادته أن تسعى لتزويد المتعلمين

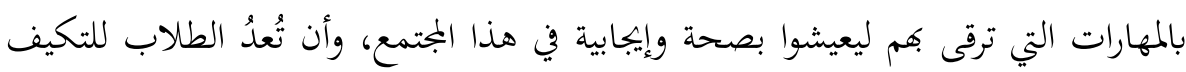
مع العالم المتغير ولمنافسة على المستويين: المحلي والعالمي في سوق العمل. لهذا تحتم على القائمين على تطوير المناهج مراعاة تضمين مهارات القرن الحادي والعشرين ضمن عناصرها بصورة تكاملية ومستمرة، حيث يرى المتخصصون أن تكامل هذه مئه المهارات بشكل مقصود ومنهجي في مناهج التعليم سوف يمكن التربويين من إنجاز العديد من الأهداف التي لم يتمكنوا من تحقيقها لسنوات طويلة مضت، ويبروون ذلك بأن هذه منه

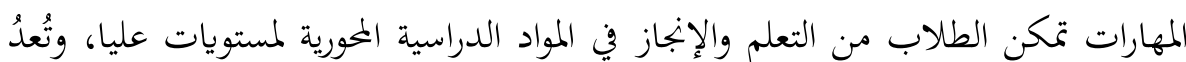
الطلاب للابتكار، والمشاركة بفاعلية في الحياة (kay, 2010).

وأكدت بعض الدراسات مثل (Sukor et al, 2010)، ( Arsada et al, 2011)، الباز (2013)، سبحي (2016)، ودراسة عيد (2019) ضرورة بناء وتطوير مناهج العلوم في ضوء مهارات القرن21، وهذا يدعو إلى ضرورة النظر للأوضاع الحالية 
لمناهج العلوم لتعرف مدى توافر مهارات القرن21 بما وتحديد مدى مساهمتها في تنمية

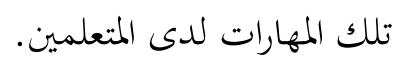

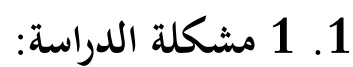

إن تحليل محتوى مقررات الكيمياء بما تتضمنه من معارف ومهارات واتحاهات يُعدا

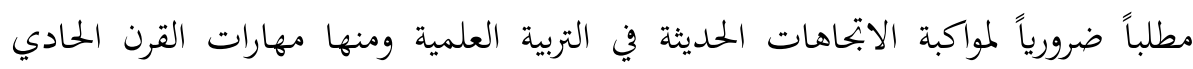

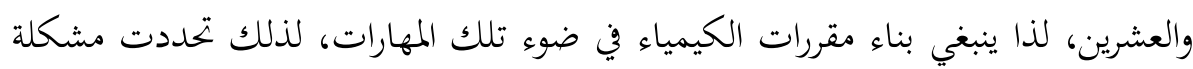
الدراسة الحالية في الإجابة عن السؤال الرئيس التالي: ما مدى تضمين مهارات القرن الحادي والعشرين في مقررات الكيمياء بالمرحلة

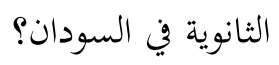
ويتفرع من السؤال الرئيس الأسئلة الفرعية الآتية:

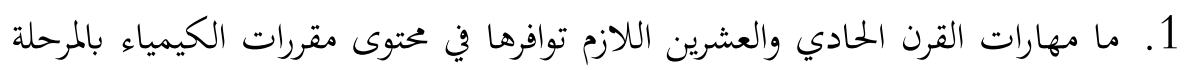

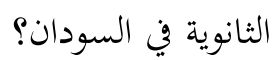

2. ما مدى تضمين مهارات القرن الحادي والعشرينفي محتوى مقررات الكيمياء للصفوف في الكوان

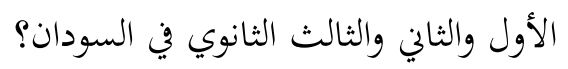

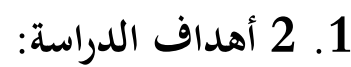

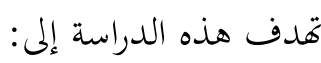
1. تحديد أهم مهارات القرن الحادي والعشرين اللازم توافرها في محتوى مقررات الكيمياء بالمرحلة الثانوية في السودان.

2. معرفة مدى تضمين كتب الكيمياء بالمرحلة الثانوية للصفوف(الأول، الثاني، الثالث) بالسودان لمهارات القرن الواحد والعشرين.

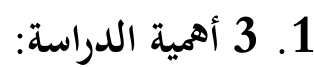
تتمثل أهمية الدراسة الحالية في: 
1. تأتي هذه الدراسة استجابة للتوجهات العالمية التي تنادي بأهمية تضمين مهارات القرن

الحادي والعشرين في مقررات التعليم عامة، ومقررات العلوم على وجه الخصوص. 2. قد تفيد مخططي ومطوري مناهج الكيمياء من حيث تزويدهم بقائمة مهارات القرن الحادي والعشرين اللازم تضمينها في محتوى مقررات الكيمياء بالمرحلة الثانوية. 3. ندرة الدراسات التي تناولت مدى تضمين مقررات الكيمياء بالمرحلة الثانوية لمهارات

القرن الحادي والعشرين- على حد علم الباحث- مما تفتح المجال لدراسات أخرى. 4. إن المشاركة في تحليل أي مقرر من مقررات التعليم العام يعد أحد المشاركات الفعالة

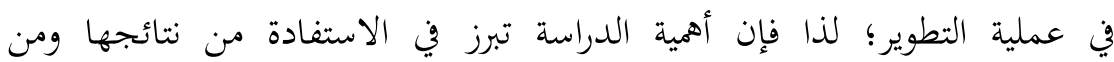
التوصيات والمقترحات التي تسهم بإذن الله في التحسين والتطوير.

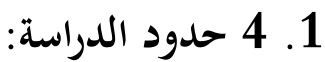
الحدود الموضوعية: تقتصر الدراسة الحالية على: - - تحليل محتوى كتب الكيمياء بالمرحلة الثانوية للصفوف(الأول، والثاني، والثالث) بالسودان للعام الدراسي 2020-2021م. - - قائمة بمهارات القرن 21 اللازم توافرها في كتب الكيمياء بالمرحلة الثانوية في السودان. الحدود الزمانية: تم تطبيق الدراسة على كتب الكيمياء بالمرحلة الثانوية التي تدرس في العام 2021-2020 1 5 1 5 مصطلحات الدراسة: يعرف الباحثان مصطلحات الدراسة إجرائياً كالآتي: مهارات القرن الحادي والعشرين: $21^{\text {st }}$ century skills هي المهارات التي يحتاجها طلاب المرحلة الثانوية، وهي: مهارات التفكير الناقد وحل

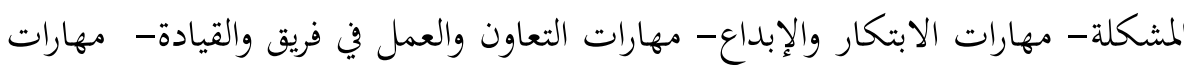
ثقافة الاتصالات والمعلومات والإعلام- مهارات ثقافة الحوسبة وتقنية المعلومات والاتصال- مهارات المهنة والتعلم المعتمد على الذات- مهارات فهم الثقافات المتعددة. 


\section{تحليل المحتوى: Content analysis} هو التحليل الوصفي الكمي لمحتويات كتب الكيمياء للمرحلة الثانوية المقررة على طلاب المرحلة الثانوية للصفوف( الأول، الثاني، الثالث) في السودان للعام 2021/2020م في لثئي ضوء مهارات القرن الحادي والعشرين اللازمة، باستخدام وحدة الموضوع كوحدة للتحليل.

\section{مقررات الكيمياء:Chemistry courses} هي المقررات التي أقرتا وزارة التربية والتعليم والمركز القومي للمناهج في السودان، وفقاً لخطة المناهج السودانية، والتي تدرس من الصف الأول حتى الصف الثالث الثانوي. 1 6 - الإطار النظري مهارات القرن الحادي والعشرين:

كانت المهارات الأساسية المطلوبة للقرن الماضي التي ينبغي للأفراد اكتسابها هي مهارات القراءة والكتابة والحساب، ولكن في ظل معطيات القرن الحادي والعشرين فقد لوحظ تنوع وتطور وسائل المعرفة والمعلومات الذي جعلها متاحة للجميع في أي زمان ومكان(الصالح، 2013: 72). ويرى الباحثان أن المهارات المطلوبة بإلحاح في القرن الحادي والعشرين، وهي: - مهارات التعلم والإبداع: وتشمل الآتي: مهارات التفكير وحل المشكلات، ومهارات الابتكار والإبداع، ومهارات الاتصال والتشارك، ومهارات الثقافة الرقمية. - مهارات الحياة والمهنة: وتشمل الآتي: مهارات المرونة والتكيف، ومهارات المبادرة والتوجيه الذاتي، ومهارات التفاعل الاجتماعي والتفاعل متعدد الثقافات، ومهارات الإنتاجية والمساءلة، ومهارات القيادة والمسؤولية. أهمية اكتساب مهارات القرن الحادي والعشرين: تكمن أهمية مهارات القرن الحادي والعشرين في أها تمكن المتعلم من التعلم والإنجاز في المواد الدراسية لمستويات عليا، كما توفر إطاراً منظماً يضمن انخراط المتعلمين في عملية التعلم، 
ويساعدهم على بناء الثقة، ويعدهم للابتكار والقيادة في القرن الحادي والعشرين والمشاركة بفعالية في الحياة المدنية(شلبي، 2014: 4)

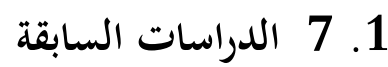

هنالك العديد من الدراسات التي تناولت مهارات القرن الحادي والعشرين، وهي مرتبة من

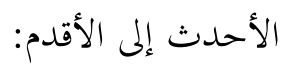

هدفت دراسة عيد (2019) إلى تحليل محتوى كتب التكنولوجيا للمرحلة الأساسية في ضوء مهارات القرن الحادي والعشرين، استخدمت الدراسة المنهج الوصفي التحليلي، وأظهرت نتائج الدراسة اتساق نتائج التحليل بين كتب التكنولوجيا للمرحلة الأساسية من حيث تقارب النسب المئوية، كما أظهرت نتائج الدراسة عدم وجود فروق ذات دلالة إحصائية عند مستوى الدلالة (0,05) تعزى لمتغير الجنس (طالب، طالبة).

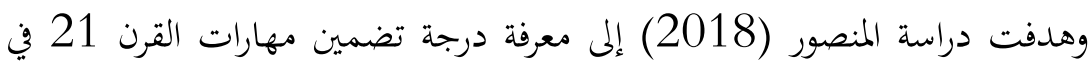
محتوى كتب العلوم لمرحلة التعليم الأساسي في الأردن، ولتحقيق هدف الدراسة استخدم المنهج الوصفي، وقد توصلت الدراسة إلى أن تضمين مهارات القرن الحادي والعشرين جاء بدرجة متوسطة.

أما دراسة حجة (2018) فهدفت إلى استقصاء مدى تضمين كتب العلوم للمرحلة الأساسية العليا للصفوف (السابع، والثامن، والتاسع) في فلسطين لمهارات القرن الحادي والعشرين الرئيسية والفرعية، وقد أشارت النتائج إلى تدني تضمين كتب العلوم لمهارات القرن الحادي والعشرين الأساسية والفرعية، وعدم تضمينها لمهارات أخرى؛ منها: استخدام التكنولوجيا، والمبادرة والتوجيه الذاتي، والقيادة والمسؤولية.

وهدفت دراسة سبحي (2016) إلى التعرف على مدى تضمين مهارات القرن 21 في مقرر العلوم المطور للصف الأول المتوسط بالمملكة العربية السعودية، وقد أظهرت نتائج الدراسة الخفاض مستوى تضمين مقررات العلوم المطورة لمهارات القرن الحادي والعشرين بنسبة بلغت (22,86\%). 
وهدفت دراسة شلبي (2014) إلى تحديد مهارات القرن الحادي والعشرين التي يمكن دججها في مناهج العلوم بمرحلة التعليم الأساسي بمصر، وقد توصلت الدراسة إلى إطار مقترح يتكون من ثلاث بجموعات من المهارات، ولكل منها مهارات أساسية وفرعية فضلاً

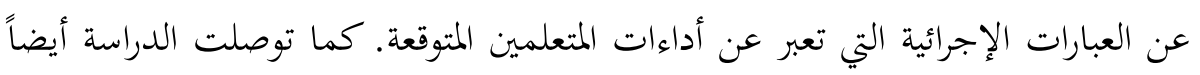

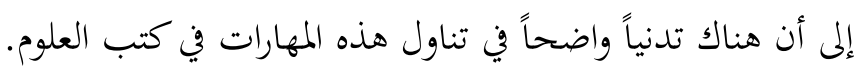
وجاءت دراسة الباز (2013) إلى تطوير منهج العلوم للصف الثالث الإعدادي في

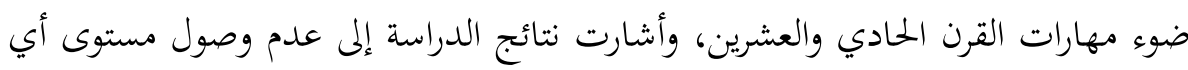
من تلاميذ الصف الثاني والثالث الإعدادي لمهارات القرن الحادي والعشرين إلى مستوى الكفاية المحدد من قبل وهو (80\%) فأكثر للنتيجة الكلية. ودراسة فوجت وروبيلين (Vooget \& Roblin, 2012) إلى مقارنة أطر المناهج الدولية في مدى تطبيقها لمهارات القرن الحادي والعشرين، وأشارت النتائج إلى وجود اتساق بين أطر المناهج الدولية حول ما هية مهارات القرن الحادي والعشرين، ولكن الممارسات والتطبيق الفعلي ما زالت بعيدة كل البعد عن التنفيذ. واهتمت دراسة ديوران (Duran, et al, 2011) بتوضيح كيف يمكن دمج مهارات القرن الحادي والعشرين في تدريس العلوم، وأشارت نتائج الدراسة إلى تحسن مشاركة وتعاون الطلاب بشكل إيجابي مماجعل بيئة التعلم أفضل، وأيضاً أن هذه الأنشطة تحقق نتائج قوية عندما تقدم خلال اليوم أو الأسبوع الأول من الفصل بئه الدراسي. وأخيراً دراسة سوكر وآخرون (Sukor, N, et al, 2010) التي هدفت إلى قياس مهارات القرن الحادي والعشرين في الكيمياء ومقارنة مستوى الطلاب وفقاً للحالة الاقتصادية والاجتماعية، وأظهرت النتائج أن الطلاب من الحالة الاقتصادية والاجتماعية العالية حققوا مستوى أعلى مقارنة بزملائهم من المستوى المنخفض. تعقيب على الدراسات السابقة: من العرض السابق يمكن توضيح أوجه استفادة الدراسة الحالية من الدراسات السابقة كما يلي: 
1. الاستفادة من الأدبيات التربوية والمنهجية العلمية لهذه الدراسات بما يخدم الدراسة

الحالية.

2. الاطلاع على الأساليب الإحصائية المستخدمة، ومعرفة المناسب منها للدراسة.

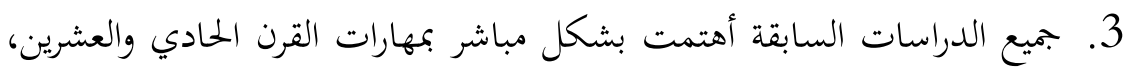

$$
\text { وأكدت على أهمية تناولها في مقررات التعليم العام. }
$$

4. أشارت عدد من الدراسات السابقة إلى وجود قصور في تناول مقررات العلوم لمهارات القرن 21 في مراحل التعليم المختلفة كدراسة شبلي(2014م)، سبحي(2016م)، و عيد(2019م) مما شجع الباحثان لإجراء الدراسة الحالية. 5. تنوعت الدراسات في المراحل التعليمية المختلفة التي تمت بها من بين مرحلة التعليم

الأساسي والمرحلة المتوسطة والثانوية والجامعية.

$$
\text { 2. } 1 \text { منهج الدراسة وإجراءاتما: }
$$

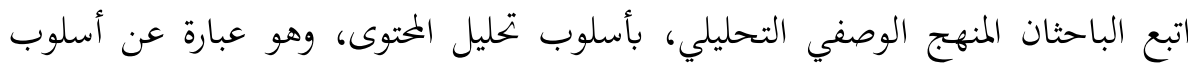
بحثي يتم تطبيقه من أجل الوصول إلى وصف كمي هادف ومنظم لمحتوى الاتصال

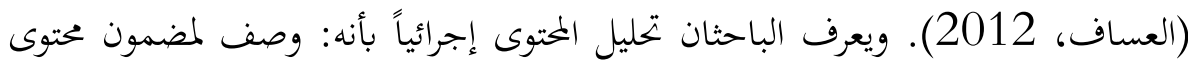
كتب الكيمياء بالمرحلة الثانوية في السودان الطبعة 2017-2018 في ضوء مهارات القرن الحادي والعشرين.

2. 2. 2 مجتمع الدراسة:

يتكون مجتمع الدراسة من كتب الكيمياء المقرةة على طلاب المرحلة الثانوية من الصف الأول حتى الصف الثالث وفقاً لآخر طبعة أقرها المركز القومي للمناهج السودانية. 2. تكونت عينة الدراسة من كتاب الكيمياء للصف الأول والثاني والثالث للعام (20202021)، وفيما يلي وصفاً للمقرر في الجدول (1). 
جدول(1)يوضح وصف مقررات الكيمياء المقررة على طلاب الصف الأول والثاني والثالث

\begin{tabular}{|c|c|c|c|c|c|}
\hline عدد الصفحات & عدد الوحدات & الصف & الطبعة & جهة التكليف & الكتاب المقرر \\
\hline 158 & 6 & الأول & 2017 & & \\
\hline 114 & 6 & الثاني & 2018/ & وزارة التربية & الكيمياء \\
\hline 400 & 9 & الثالث & & والتعليم & \\
\hline
\end{tabular}

2. - 2. 3

اعتمدت الدراسة لتحليل محتوى كتب الكيمياء المقررة على طلاب الصف الأول والثاني والثالث الثانوي على أداة تحليل محتوى، والتي تضمنت قائمة مهارات القرن الحادي والعشرين التي أعدها الباحثان بعد الاطلاع على الأدب التربوي والدراسات السابقة في هذا المجال قد بينت هذه الأداة باتباع الخطوات الآتية: 1) الصورة الأولية للقائمة: تم بناء قائمة بمهارات القرن الحادي والعشرين بعد الاطلاع على الأدب التربوي والدراسات السابقة ذات العلاقة بالدراسة، وقد تكونت القائمة بصورةا الأولية من (50) مؤشر موزعين على سبعة محاور رئيسية.

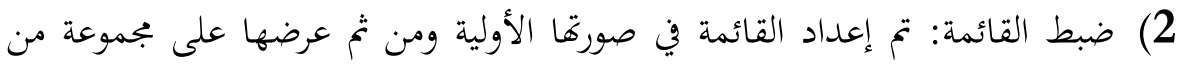
المحكمين المختصين في بجال المناهج وطرق تدريس العلوم وبعض موجهي ومعلمي الكيمياء. ولتحديد مدى أهمية تضمين المحاور الرئيسية والفرعية في كتب الكيمياء وكذلك حذف ما هو غير مناسب وإضافة ما هو مناسب وإعادة صياغة لبعض المؤشرات، وقد أدت عملية التحكيم إلى إجراء بعض التعديلات وذلك من خلال حذف بعض المؤشرات وإضافة مؤشرات أخرى وإعادة صياغة بعض المؤشرات. 3) الصورة النهائية للقائمة: بعد إجراء التعديلات التي أشار إليها المحكمون تم وضع

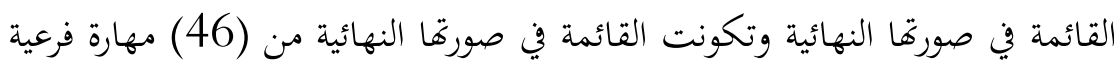
موزعين على سبعة بجالات رئيسة، وهي: (التفكير الناقد وحل المشكلات- الابتكار 
والإبداع- التعاون والعمل في فريق والقيادة- ثقافة الاتصالات والمعلومات والإعلامثقافة الحوسبة وتقنية المعلومات والاتصال- المهنة والتعلم المعتمد على الذات- فهم

$$
\text { الثقافات المتعددة). }
$$

4) إجراءات التحليل:تمت عملية تحليل المحتوى في ضوء مهارات القرن 21 كالتالي: أ. الهدف من التحليل: تحديد مدى تناول كتب الكيمياء بالمرحلة الثانوية في السودان مهارات القرن الحادي والعشرين الرئيسية والفرعية التي تم تضمينها في القائمة التي تم

$$
\text { إعدادها سابقاً في هذه الدراسة. }
$$

ب. فئة التحليل: تم تحليل محتوى كتب الكيمياء بالمرحلة الثانوية في ضوء مهارات القرن

$$
\text { الحادي والعشرين التي تم تحديدها سابقاً. }
$$

ج. وحدة التحليل: اعتمد الباحثان الموضوع كوحدة تحليل حيث قام الباحث بتحليل المحتوى بناء على توافر مهارات القرن الحادي والعشرين في كل موضوع. د. عينة التحليل: تمثلت عينة التحليل في كتب الكيمياء المقررة على طلاب الصف الأول والثاني والثالث الثانوي للعام(2020-2021).

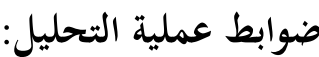
لأجل ضبط عملية التحليل تم مراعاة الضوابط المقترحة من قبل السادة المحكمين، وهي كما يلي: - - تم التحليل في إطار المحتوى العلمي للكتب، مع استبعاد الغلاف ومقدمة الكتاب والفهارس. - اشتمل التحليل على أسئلة اختبار الوحدة وهاية كل وحدة والأنشطة الواردة في المحتوى. 
جدول(2) توصيف كتب الكيمياء المقررة على طلاب الصف الأول والثاني والثالث الثانوي الثان الثان

\begin{tabular}{|c|c|c|c|}
\hline عدد الصفحات & عنوان الوحدة & الوحدة & الصف \\
\hline 35 & التركيب الذري للمادة & الأولى & \multirow{6}{*}{ الأول } \\
\hline 38 & الروابط الكيميائية & الثانية & \\
\hline 14 & المعادلات الكيميائية & 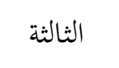 & \\
\hline 17 & التفاعلات الكيميائية & 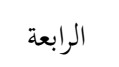 & \\
\hline 36 & الحساب الكيميائي & الخامسة & \\
\hline 15 & قوانين الإتحاد الكيميائي & السادسة & \\
\hline 22 & الترتيب الدوري للعناصر & الأولى & \multirow{6}{*}{ الثاني } \\
\hline 13 & فلزات المجموعة الأولى & الثانية & \\
\hline 26 & الكيمياء العضوية & 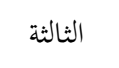 & \\
\hline 18 & النتروجين & 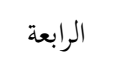 & \\
\hline 19 & الهالوجينات & الخامسة & \\
\hline 11 & العناصر الانتقالية & السادسة & \\
\hline 85 & $\begin{array}{c}\text { الكيمياء العضوية والمشتقات الهيدروكربونية } \\
\text { وتطبيقاتما }\end{array}$ & 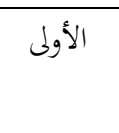 & \multirow{9}{*}{ الثالث } \\
\hline 19 & الأماض والقواعد & الثانية & \\
\hline 43 & التحليل الكيميائي الكيفي & 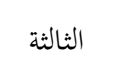 & \\
\hline 68 & التحليل الكيميائي الحجمي & 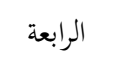 & \\
\hline 29 & الطاقة في الثفاعلات الكيميائية & الخامسة & \\
\hline 26 & سرعة وآلية التفاعلات الكيميائية & السادسة & \\
\hline 30 & مبادئ الاتزان الكيميائي & السابعة & \\
\hline 59 & الكيمياء الكهربية & 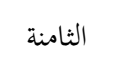 & \\
\hline 34 & الكيمياء النووية & التاسعة & \\
\hline
\end{tabular}




\section{صدق أداة التحليل:}

لمعرفة صدق أداة التحليل الذي يقصد بها "مدى تحقيق الأداة للغرض الذي أعدت من أجله، فتقيس ما وضعت لقياسه ويعتمد مدى تمثيل بنود المقياس تمثيلاً سليماً للمجال

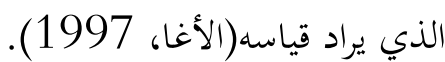

ولقد تم الاستدال على صدق أداة التحليل من خلال صدق القائمة التي استدل على صدقها من خلال صدق المحكمين، فقد عرضت القائمة على مجموعة من المحكمين المختصين في المناهج وطرق تدريس العلوم وبعض موجهي ومعلمي مادة الكيمياء، وذلك لإبداء آرائهم في إمكانية تحليل محتوى الكيمياء لمعرفة مدى توافر مهارات القرن الحادي والعشرين، باستخدام هذه الأداة ولقد أشار المحكمون إلى صلاحية الأداة للاستخدام في عملية التحليل من حيث البنود والمقياس مع إضافة بعض التعديلات على القائمة.

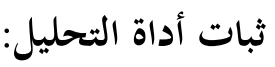

بعد أن تم التأكد من صدق القائمة تم معرفة ثبات التحليل ويقصد بالثبات "استقرار نتائج القياس إذا ما أعيد تطبيقه على نفس العينة" (عدس، 2013). وتم التأكد

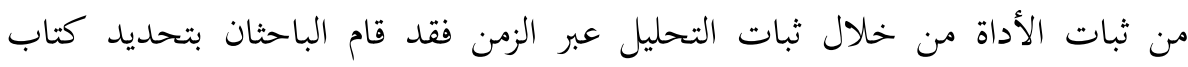

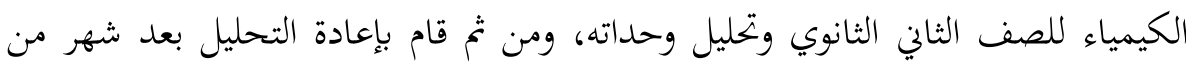
التحليل الأول، ومن ثم حسب نسبة الاتفاق بين التحليلين الأول والثاني باستخدام معادلة هولستي للثبات، الجدول التالي يوضح نقاط الاتفاق والاختلاف في التحليلين. 
جدول(3) نقاط الاتفاق والاختلاف في نتائج تحليل وحدات كتاب الكيمياء للصف الثاني

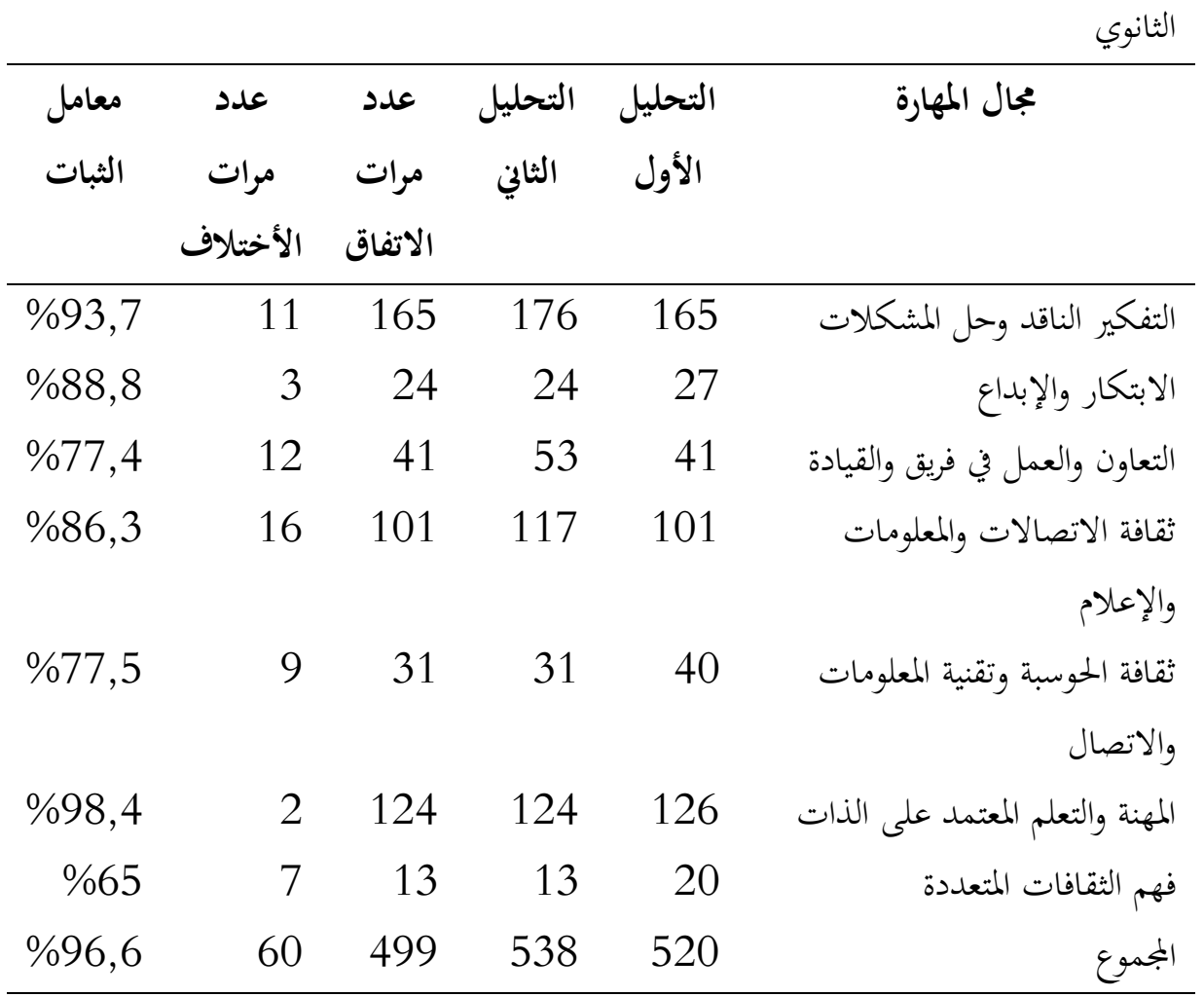

ولتأكد من ثبات أداة التحليل تم استخدام معادلة هولستي والتي أظهرت أن معامل الثبات بلغت قيمته(96,6\%) وهي نسبة عالية ومطمئنة لاستخدام أداة تحليل المحتوى.

3.

3. 1 النتائج المتعلقة بالسؤال الأول ومناقشتها:

ينص السؤال الأول على ما يلي: ما مهارات القرن الحادي والعشرين الواجب

توافرها في محتوى كتب الكيمياء بالمرحلة الثانوية؟ للإجابة عن هذا السؤال قام الباحثان بالاطلاع والبحث في الأدب التربوي، والدراسات السابقة والأبحاث، والكتب ذات العلاقة بمهارات القرن الحادي والعشرين، مع الاطلاع على بعض التجارب والمشاريع العالمية والعربية ذات العلاقة؛ ومنها: عالمياً منظمة التعلم والقياس للقرن القرن الحادي والعشرين 
(ATC 21ST)، ومنظمة الشراكة من أجل مهارات القرن الحادي والعشرين بالولايات المتحدة (Partnership 21 $21^{\text {st }}$ century Skills of US)، والمختبر التربوي للإقليم الشمالي المركزي (NCREL,2003)، ومهارات الجمعية الدولية للتكنولوجيا في التعليم والاتحاد الأروبي، أما عربياً فقد قام الباحثان بالاطلاع على إطار (ISTE, 2013) المنظمة العربية للتربية والثقافة والعلوم "ألكسو"، ومن الدراسات السابقة ذات العلاقة

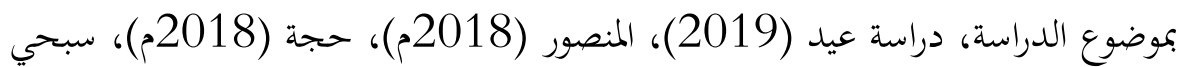
Vooget \& (2016)، حسن (2015م)، الباز (2013م)، فوجت وروبيلين (2013)

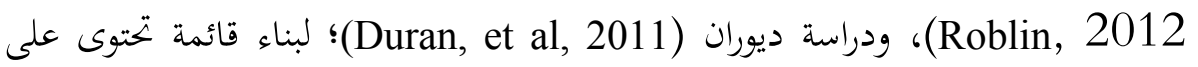
مهارات القرن الحادي والعشرين الواجب توافرها فيكتب الكيمياء بالمرحلة الثانوية، ودراء واشتملت القائمة على (46) مهارة فرعية موزعين على سبعة مجالات رئيسة كما يلي:

جدول (4) قائمة بمهارات القرن الحادي والعشرين الواجب توافرها في محتوى مقررات الكيمياء بالمرحلة الثانوية بالسودان

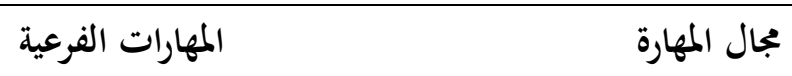

- يوجه محتوى مقررات الكيمياء المتعلم لكتابة عبارات تبين رأيه. - يوجه محتوى مقررات الكيمياء المتعلم لتفسير الأفكار وتوضيحهاء. - يتضمن محتوى الكيمياء مواقف لتنمية مهارات التخاذ القرار. التفكير الناقد وحل يتضمن محتوى الكيمياء تحليل بدائل وجهات النظر. المشكلات ينمي مهارات تفسير المعلومات والآراء والأحداث. - يتضمن فرضاً لحل المسائل بشكل مستقل. - يعطي فرصة للحكم على الإجابات المختلفة. - يتضمن أنواعاً يختلفة من المشكلات والمواقف غير الماتحات المألوفة. - يتضمن أسئلة توضح وجهات النظر المتنوعة. 
- يقدم المختوى طرقاً مختلفة لابتكار الأفكار (كالعصف الذهني). الابتكار والإبداع - يحث المحتوى على بناء الأفكار وتوسيعها.

- يحث المحتوى على إضافة تفاصيل جديدة ومتنوعة لفكرة ما. - يطلب تفسيرات غير مألوفة للبيانات والأشكال. - يحث على تنظيم المعلومات وفق أفكار جديدة. - يعزز مقررات الكيمياء الاتحاهات الإيجابية نحو العمل الجماعي. - يوجه إلى استثمار نقاط القوة في الآخرين. التعاون والعمل في فريق والقيادة - يتضمن مواقف تعليمية بالمشروعات الجماعية. - يركز على قضايا المجتمع ومصالحه. - يتضمن مواقف تحث على المبادرة والقيادة. - يوجه السلوك الإنساني نحو الاستقامة. - ينمي المشاركة والتعاون بفاعلية. - يوجه السلوك بأسلوب مهني.

ثقافة الاتصالات - تتضمن مقررات الكيمياء مواقف لتنمية مهارات الاتصال الشفهي. والمعلومات والإعلام - يتضمن محتوى مقررات الكيمياء مواقف لتنمية مهارات الاتصال المكتوب. - يساعد المحتوى على الوصول للمعلومات بكفاءة الوقت وفاعلية المصادر. - يحث على استخدام المعلومات بشكل دقيق. - ينمي تقويم المعلومات تقويماً نقدياً. - يوجه المحتوى لما ينشر في الإعلام والاستفادة منه. ثقافة الحوسبة وتقنية - يحث على استخدام وسائل وتقنيات إعلامية متعددة. المعلومات والاتصال - يحث على إصدار الأحكام على فاعلية الوسائل والتقنيات الإعلامية المتعددة.

- يعزز محتوى مقررات الكيمياء الاتحاهات الإيجابية نحو التقنية. 
- يتضمن مواقف تتطلب توظيف استخدام التقنيات الحديثة. - يتضمن مواقف لاستخدام التقنيات الرقمية للوصول للمعلومات وإدارتا ودمجها وتقويمها. - يجث محتوى الكيمياء على إصدار الأحكام على نوعية مصادر المعلومات.

$$
\text { - ينمي تكييف المتعلم لأدوار ومسؤوليات متنوعة. }
$$
المهنة والتعلم المعتمد - يتضمن مواقف تعليمية بمشروعات تزداد تعقيداً بالتدريج.

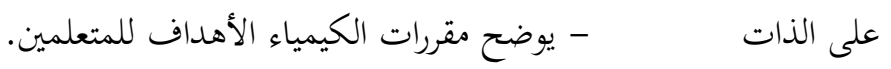
- يوجه محتوى مقررات الكيمياء لإدارة المشاريع بكفاءة. - يحفز المتوى المتعلمين على التساؤل الذاتي. - يعطي فرصة لتجاوز متطلبات المنهج إلى استكشاف وتوسيع التعلم الشخصي. - ينمي الشعور بالمسؤولية وتحمل النتائج. - يعز الابحاهات الإيجابية نحو الثقافات الأخرى. - يتضمن مواقف لتنمية مهارات التفاعل مع الآخرين(كالإصغاء

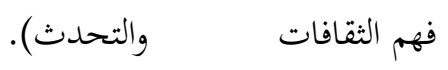

- يشجع الاستجابة للقيم الاجتماعية المختلفة. الأخرى - يشير إلى ما يميز ثقافات البلدان المختلفة.

\section{2 النتائج المتعلقة بالسؤال الثاني ومناقشتها}

للإجابة عن السؤال الثاني الذي نصه "ما مدى تضمين مهارات القرن الحادي والعشرين في محتوى مقرر الكيمياء للصف الأول الثانوي بالسودان؟ تم تحليل كتاب الكيمياء للصف الأول الثانوي في ضوء مهارات القرن الحادي والعشرين، ورصد تكرارها، وكانت النتائج كما يظهر في جدول (5). 
جدول(5) نتائج تحليل كتاب الكيمياء للصف الأول الثانوي في ضوء مهارات القرن الحادي والعشرين

نتائج تحليل كتاب الكيمياء للصف الأول الثانوي

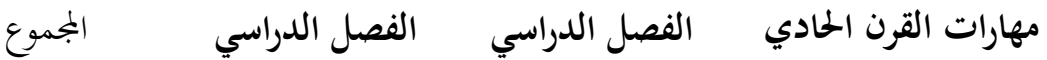

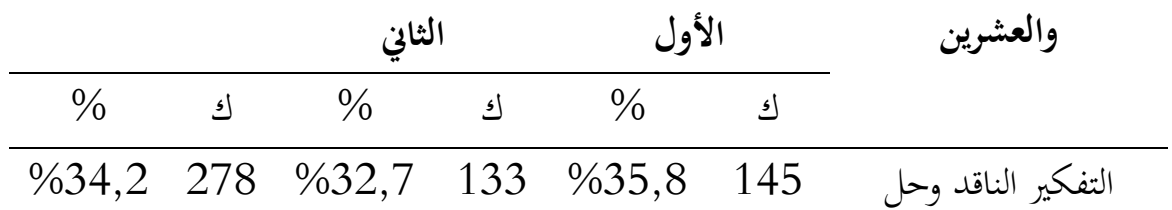

الابتكار والإبداع

التعاون والعمل في فريق

ثقافة الاتصالات والقيادة والمعلومات والإعلام

ثقافة الحوسبة وتقنية المعلومات والاتصال

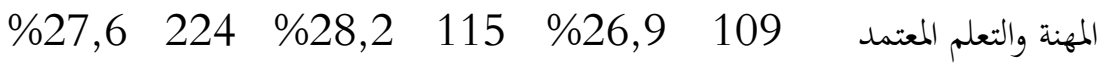
على الذات

فهم الثقافات المتعددة المجموع 100 $812 \% 100 \quad 405 \quad \%$

يتضح من جدول (5) تضمين جميع مهارات القرن الحادي والعشرين في كتاب الكيمياء للصف الأول الثانوي، وذلك بواقع (812) تكراراً، توزعت بين الفصلين الدراسين الأول والثاني، وأن المهارات الرئيسة السبع قد تضمنها محتوى كتاب الكيمياء للصف الأول الثانوي بنسب متفاوتة، وقد جاء في المرتبة الأولى مهارات "التفكير الناقد

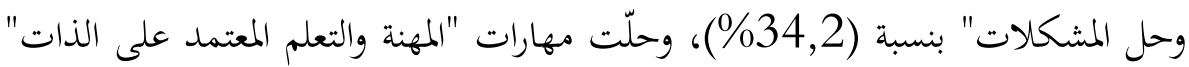


بنسبة توافر بلغت (27,6\%) في المرتبة الثانية، بينما جاءت مهارات " ثقافة الاتصالات والمعلومات والإعلام" بنسبة توافر بلغت (24,8\%) في المرتبة الثالثة، وجاءت باقي المهارات الرئيسة بنسب متدنية فقد جاء في المرتبة الرابعة مهارات "ثقافة الحوسبة وتقنية

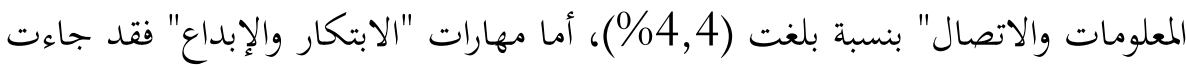
بنسبة بلغت (4,1\%)، بينما حلّت مهارتي "التعاون والعمل في فريق والقيادة" و "فهم بهم الثقافات المتعددة" في المرتبتين الأخيرتين بنسب بلعت (4,4\%) و(1,5\%) على التوالي. يتضح مما سبق وجود تدني ملحوظ في تناول مقرر الكيمياء للصف الأول الثانوي

لمهارات القرن الحادي والعشرين، حيث اتفقت نتائج الدراسة الحالية مع نتائج الدراسات السابقة في مناهج العلوم على المستويين العربي والعالمي كدراسة حجة (2018م)، سبحي

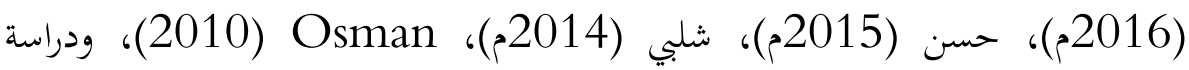
Sukor الحادي والعشرين، وقصوراً في تناولها لمهارات هذا القرن، محا أغفلت دورها البارز في إعداد

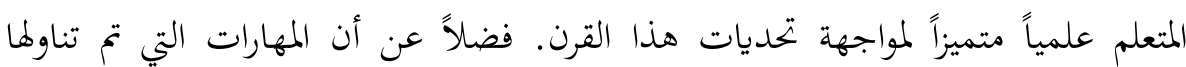
بالمتوى تتسم بالمعالجة السطجية حيث جاء معظمها عرضياً وغير موظف بطريقة علمية سليمة.

\section{3 النتائج المتعلقة بالسؤال الثالث ومناقشتها}

للإجابة عن السؤال الثالث الذي نصه "ما مدى تضمين مهارات القرن الحادي والعشرين في محتوى مقرر الكيمياء للصف الثاني الثانوي بالسودان؟ تم تحليل كتاب الكيمياء للصف الثاني الثانوي في ضوء مهارات القرن الحادي والعشرين، ورصد تكرارها،

$$
\text { وكانت النتائج كما يظهر في جدول (6). }
$$


جدول (6) نتائج تحليل كتاب الكيمياء للصف الثاني الثانوي في ضوء مهارات القرن الحادي والعشرين

نتائج تحليل كتاب الكيمياء للصف الثاني الثانوي

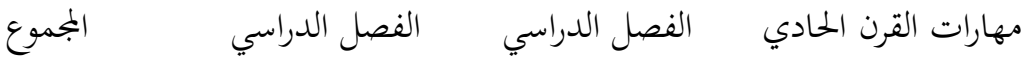

\begin{tabular}{|c|c|c|c|c|c|c|}
\hline & & \multicolumn{2}{|c|}{ الثاني } & \multicolumn{2}{|c|}{ الأول } & \multirow[t]{2}{*}{ والعشرين } \\
\hline$\%$ & 5) & $\%$ & 5) & $\%$ & s) & \\
\hline 32,2 & 341 & 32,7 & 176 & 31,7 & 165 & التفكير الناقد وحل \\
\hline
\end{tabular}

المشكلات

الابتكار والإبداع

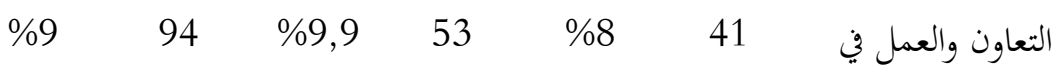

فريق والقيادة

ثقافة الاتصالات

والمعلومات والإعلام

ثقافة الحوسبة وتقنية

المعلومات والاتصال

المهنة والتعلم المعتمد

على الذات

فهم الثقافات المتعددة

المجموع

يتضح من جدول (6) تضمين جميع مهارات القرن الحادي والعشرين في كتاب

الكيمياء للصف الثاني الثانوي، وذلك بواقع (1058) تكراراً، توزعت بين الفصلين

الدراسين الأول والثاني، وأن المهارات الرئيسة السبع قد تضمنها محتوى كتاب الكيمياء للصف الثاني الثانوي بنسب متفاوتة، وقد جاء في المرتبة الأولى مهارات "التفكير الناقد وحل المشكلات" بنسبة (32,2\%)، وحلّت مهارات "المهنة والتعلم المعتمد على الذات" 
بنسبة توافر بلغت (23,6\%) في المرتبة الثانية، بينما جاءت مهارات " ثقافة الاتصالات والمعلومات والإعلام" بنسبة توافر بلغت (20,6\%) في المرتبة الثالثة، وجاءت باقي المهارات الرئيسة بنسب متدنية فقد جاء في المرتبة الرابعة مهارات "التعاون والعمل في فريق

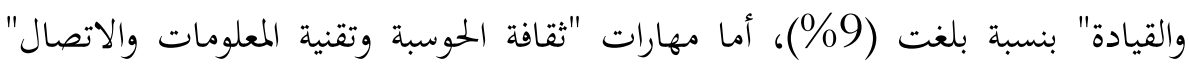
فقد جاءت بنسبة بلغت (\%6,7)، بينما حلّت مهارتي "الابتكار والإبداع" و "فهم الثقافات المتعددة" في المرتبتين الأخيرتين بنسب بلعت (4,8\%) و (1,1\%) على التوالي.

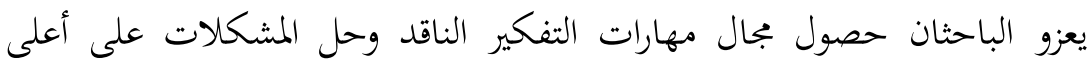
نسبة تضمين يرجع إلى طبيعة مادة الكيمياء والدور الذي تقوم به في تنمية مهارات التفكير الناقد وحل المشكلات التي تواجه طلاب المرحلة الثانوية. تتفق نتيجة الدراسة الحالية مع نتائج دراسة عيد (2019م)، سبحي (2016م)،

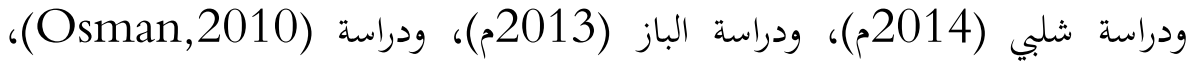
ودراسة (Sukor, 2010)، ويمكن تفسير هذه نتائج الدراسة بأن معايير بناء مناهج

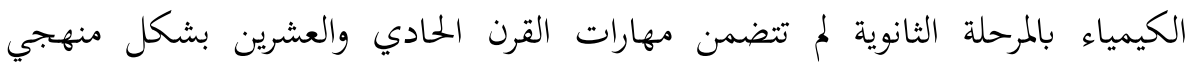

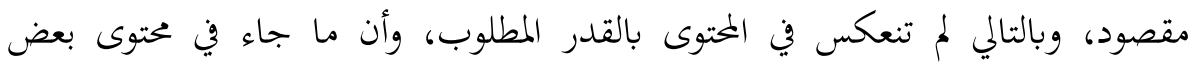
الدروس تم تناوله بشكل عرضي غير مقصود تم طرحه نتيجة لاجتهاد مؤلفي هذه الكتب في تحقيق بعض المهارات، وأن كان في بجمله ضعيف.

\section{4 النتائج المتعلقة بالسؤال الرابع ومناقشتها}

للإجابة عن السؤال الثاني الذي نصه "ما مدى تضمين مهارات القرن الحادي والعشرين في محتوى مقرر الكيمياء للصف الثال الثانوي بالسودان؟ تم تحليل كتاب الكيمياء للصف الثالث الثانوي في ضوء مهارات القرن الحادي والعشرين، ورصد تكرارها، وكانت النتائج كما يظهر في جدول (7). 
جدول(7) نتائج تحليل كتاب الكيمياء للصف الثالث الثانوي في ضوء مهارات القرن

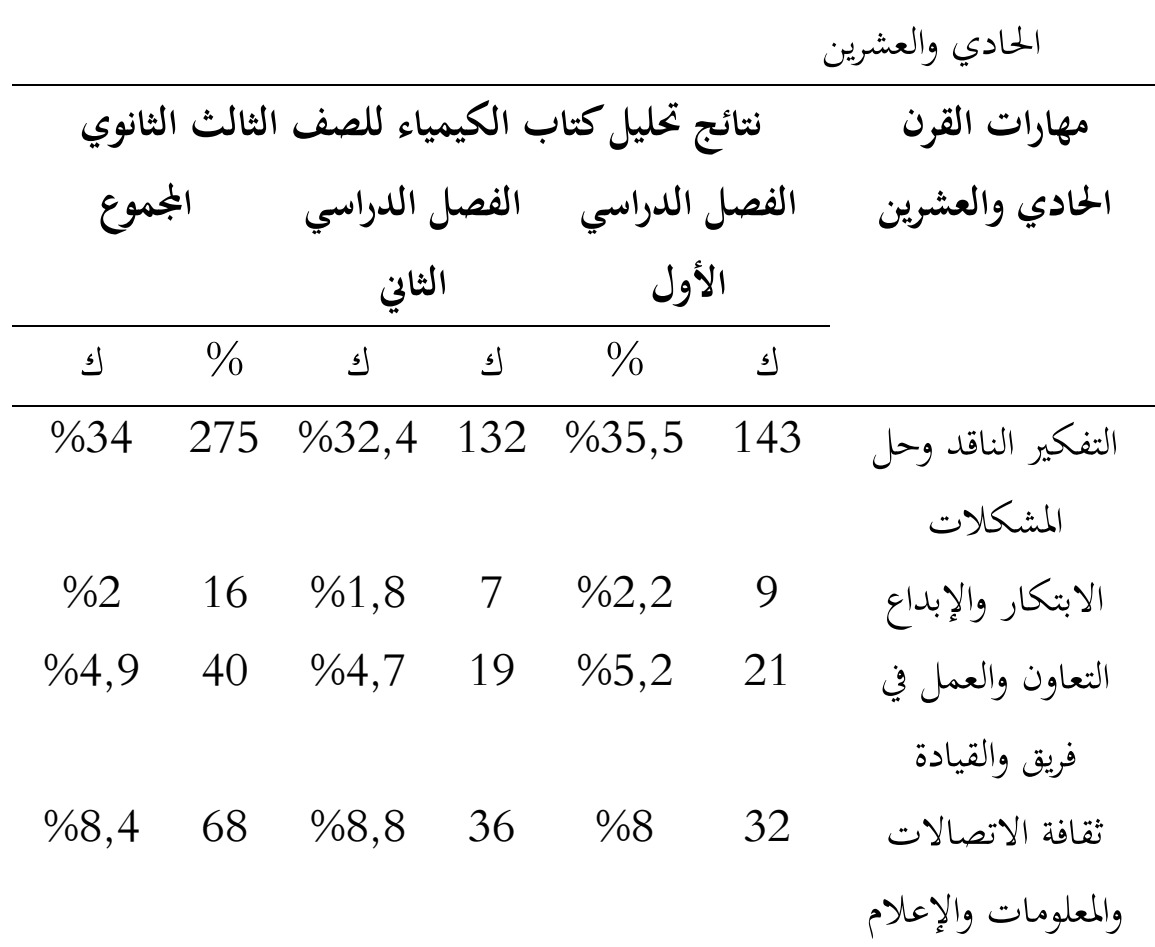

ثقافة الحوسبة وتقنية 16 المعلومات والاتصال

المهنة والتعلم المعتمد

$$
\text { على الذات }
$$

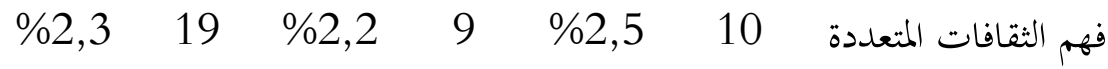

المجموع

يتضح من جدول (7) تضمين جميع مهارات القرن الحادي والعشرين في كتاب

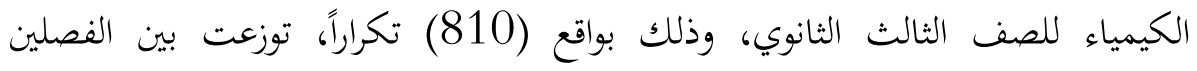

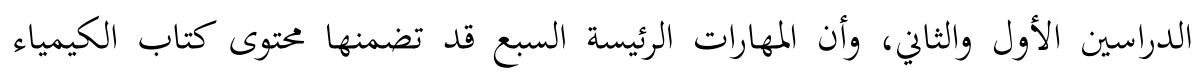
للصف الثالث الثانوي بنسب متفاوتة، وقد جاء في المرتبة الأولى مهارات "المهنة والتعلم 
المعتمد على الذات" بنسبة (44,2\%)، وحلّت مهارات "التفكير الناقد وحل المشكلات" بنسبة تضمين بلغت (34\%) في المرتبة الثانية، وجاءت باقي المهارات الرئيسة بنسب

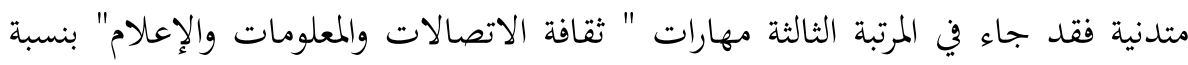
توافر بلغت (8,4\%) ، بينما جاءت مهارات "التعاون والعمل في فريق والقيادة" بنسبة

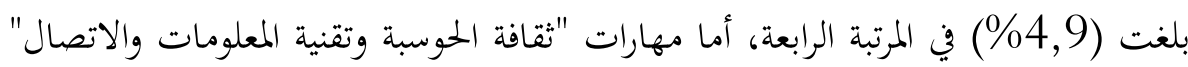
فقد جاءت بنسبة بلغت (4,2\%)، بينما حلّت مهارتي "فهم الثقافات المتعددة" و وركات "الابتكار والإبداع" في المرتبتين الأخيرتين بنسب بلعت (2,3\%) و و(2\%) على التوالي. ويعزو الباحثان تدني مستوى تضمين مهارات القرن الحادي والعشرين في محتوى مقررات الكيمياء بالمرحلة الثانوية إلى أهما لم تتضمن بشكل منهجي، وهو ما اتفقت عليه

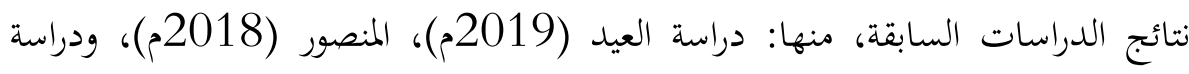
حجة (2018م)، سبحي (2017م)، شبي (2014م)، ودراسة الباز (2013م)، حيث أشارت مجتمعه إلى وجود تدلي واضح في تناول مهارات القرن الحادي والعشرين، وأن مستوى تضمين مهارات القرن الحادي والعشرين لم يصل إلى المستوى المطلوب.

4. الحخلاصة

$$
\text { يمكن تلخيص أهم النتائج فيما يلي: }
$$

1. إعداد قائمة بمهارات القرن الحادي والعشرين التي ينبغي أن تتوافر في محتوى مقررات فيمات

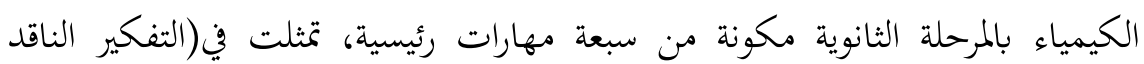
وحل المشكلات- الابتكار والإبداع- التعاون والعمل في فريق والقيادة- ثنافة تئه الاتصالات والمعلومات والإعلام- ثقافة الحوسبة وتقنية المعلومات والاتصال- المهنة

$$
\text { والتعلم المعتمد على الذات- فهم الثقافات المتعددة). }
$$

2. انخفاض مستوى تضمين مقررات الكيمياء بالمرحلة الثانوية لمهارات القرن الحادي والعشرين بلغت أعلى نسبة (44,2\%) وأدنى نسبة تضمين بلغت (1,5\%). 


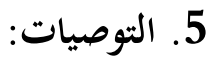

بناءً على النتائج التي تم التوصل إليها يمكن الخروج بالتوصيات التالية:

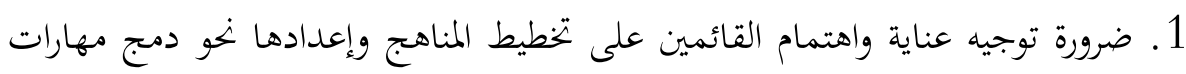
القرن الحادي والعشرين في الخطط الدراسية لجميع المراحل التعليمية. 2. وضع معايير لبناء مناهج الكيمياء بالمرحلة الثانوية بحيث تتضمن مهارات القرن الحادي والعشرين بشكل منهجي مقصود يحقق مبدأ التكامل والاستمرارية. 3. ضرورة تدريب معلمي الكيمياء على مهارات القرن الحادي والعشرين.

\section{المراجع}

الأغا، إحسان (1997م). البحث التربوي عناصره منهاجه أدواته، ط3، مطبعة مقداد، غزة.

الباز، مروة محمد (2013م). تطوير منهج العلوم للصف الثالث الإعدادي في ضوء

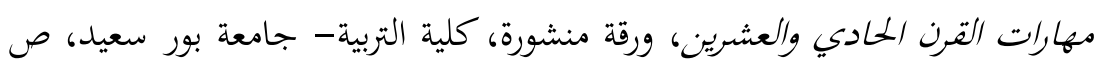

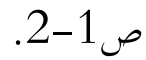
حجة، حكم (2018م). مدى تضمين كتب العلوم للمرحلة الأساسية العليا لمهارات القرن الحادي والعشرين، دراسات العلوم التربوية، 45(3)، 163-178.

حسن، ياسمين (2015م). تقويم محتوى وأنشطة مناهج الدراسات الاجتماعية بالمرحلة

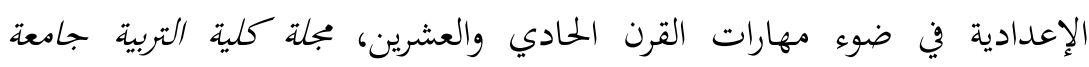

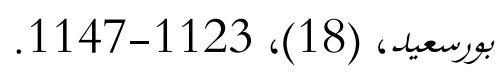

سبحي، نسرين بنت حسن (2016م). مدى تضمين مهارات القرن الحادي والعشرين في مقرر العلوم المطور للصف الأول المتوسط بالمملكة العربية السعودية، مجلة العلوم التربوية، 1(1)، 15-16. 
السعيد، سعيد محمد والماضي، عبدالرمن بن إبراهيم (2013م). مشكلات تدريس مناهج

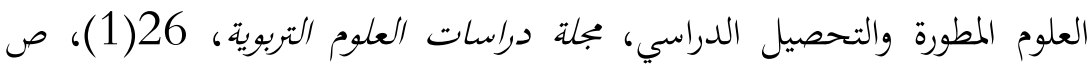

شلبي، نوال(2014م). إطار مقترح لدمج مهارات القرن الحادي والعشرين في مناهج

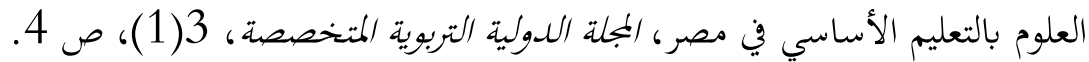

الصالح، بدرية عبدالله (2013م). مهارات القرن الحادي والعشرين التعلم للحياة في زمنا، الرياض، جامعة الملك سعود.

عدس، عبدالرحمن (2013م). مبادئ الإحصاء في التربية وعلم النفس ، ط1، دار الفكر،

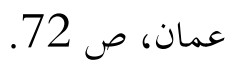

العساف، صالح (2012م). المدلخل إلى البحث في العلوم السلوكية، الرياض، العبيكان. عيد، سمية إبراهيم سلام شيخ (2019م). تحليل كتب التكنولوجيا للمرحلة الأساسية في ضوء مهارات القرن الحادي والعشرين ومادى اكتساب طلبة الصف العاشر لها،

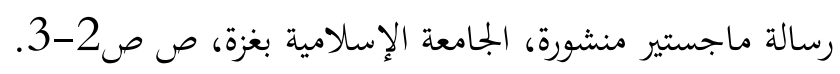

المنصور، عرين سليمان (2018م). درجة تضمين كتب العلوم لمرحلة التعليم الأساسي في الأردن لمهارات القرن الحادي والعشرين(رسالة ماجستير غير منشورة)، جامعة آل

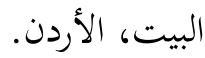

\section{ثانيا: المراجع باللغة الانجليزية}

Arsada, N, Osmana, K \& Tuan, M. (2011). Instrument development for $21^{\text {st }}$ century skills in Biology. Procedia social and Behavioral science (15), 1470-1474.

Duran, E., Yaussy, D., \& Yaussy, L. (2011). Race to the future: Integrating $21^{\text {st }}$ century skills into science instruction. Science 
Activities: Classroom Projects and Curriculum Ideas, 48(3), 98106.

Kay, K. (2010). 21 ${ }^{\text {st }}$ century skills: why they matter, what they are, and How we get there. $21^{\text {st }}$ century skills: Rethinking how students learn. Edited by, James Bellance, Ron Brandt. Bloomington, IN: Solution Tree Press, xiii-xxxi.

Osman, K, and Sho T.M.T and Arsad, N.M (2010). Development and validation of the Malaysian $21^{\text {st }}$ century skills instrument $(\mathrm{m}-21$ CSI) for science student WCLTA 2010, Procedia social and behavioral sciences, vol 9, 599-603.

Sho, T.M.T., Arsad, N. \& Osman. K. (2010). The relationship between $21^{\text {st }}$ century skills on student's attitude and perception towards physic. Social and Behavioral Science (7), 546-554.

Sukor N.M and Osman K. and Abdullah (2010). Students achievement of Malaysian $21^{\text {st }}$ century skills in chemistry, WALTA 2010. Procedia social and behavioral sciences, vol 9, 1256-1260.

Voogt, J, \& Roblin, N. P. (2012). A comparative analysis of international frameworks for $21^{\text {st }}$ century competences: Implications for national Curriculum policies. Journal of Curriculum Studies, 44(3), 299-321. 